\title{
THE CONNECTION PATTERNS OF TWO COMPLETE BINARY TREES*
}

\author{
F. R. K. CHUNG† AND F. K. HWANG $\dagger$
}

\begin{abstract}
We consider the class of channel graphs which can be viewed as compositions of two copies, right and left, of a complete binary tree with terminal nodes of the right tree connected to distinct terminal nodes of the left tree. We study the connection patterns of the two binary trees to minimize the blocking probability of the resulting channel graphs. We show that the connection patterns given by Ikeno are not optimal in general and in fact no optimal connection patterns exist for such graphs with more than 9 stages. We present new connection patterns which uniquely possess certain optimal properties.
\end{abstract}

1. Introduction. We consider the class of graphs which consist of two copies, right and left, of a complete binary tree with terminal nodes of the right tree connected to distinct terminal nodes of the left tree. If the complete binary trees have $n$ levels and $2^{n-1}$ terminal nodes, then there are $\left(2^{n-1}\right)$ ! possible ways to connect these two trees, though some of them might be isomorphic. In Fig. 1 there are two nonisomorphic graphs, each of which is formed by combining two binary trees having three levels. In general such a graph can be viewed as a $2 n$-stage network having the roots of the two complete binary trees as the source and sink of the network. We call such a graph a binary channel graph or a binary graph. We note that a binary graph is a special case of multistage channel graphs, also called linear graphs, which are often used in studying the blocking performance of switching networks [3]. A channel graph can be viewed as the union of all paths connecting a specified pair of input and output terminals in a switching network. In an $m$-stage channel graph, all vertices are partitioned into a sequence of $m$ subsets, called stages; edges, called links, exist only between vertices in adjacent stages. The links between stage $i$ and stage $i+1$ are referred to as the $i$ th stage links, and we assume that each $i$ th stage link has probability $p_{i}$ of being occupied. The vector $\left(p_{1}, \cdots, p_{m-1}\right)$ is called the link occupancies of the channel graph. The blocking probability of a channel graph is defined to be the probability that every path from the source to the sink contains at least one occupied edge. Two channel graphs of the same number of stages can be compared in the following way. We say one channel graph is superior to the other if the blocking probability of the former never exceeds that of the latter for any given link occupancies. A $2 n$-stage binary graph is said to be optimal if it is superior to any other $2 n$-stage binary graph.

The problem of determining the connection pattern of the two binary trees to minimize the blocking probability of the resulting binary graph is not only interesting on its own right but also useful in designing effective switching networks (see [3]). Ikeno [1] investigated this problem and suggested the following simple and elegant connection: Assign binary numbers to terminal nodes of the right and left trees in an orderly fashion and connect two terminal nodes such that the digits of the corresponding binary numbers are inversions of each other. For example, the connections in the graph in Fig. $1(\mathrm{~b})$ are $(0,0)$ to $(0,0),(0,1)$ to $(1,0),(1,0)$ to $(0,1)$ and $(1,1)$ to $(1,1)$.

It can be easily verified that the Ikeno graph is optimal for $n=3$. However, the optimality for Ikeno graphs for $n>3$ has not been previously determined in the past,

\footnotetext{
* Received by the editors October 4, 1979.

$\uparrow$ Bell Laboratories, Murray Hill, New Jersey 07974.
} 


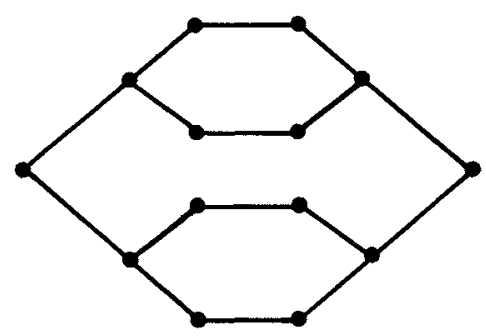

(a)

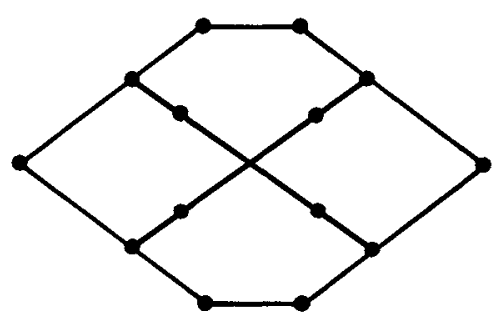

(b)

FIG. 1

though there has been a general willingness to believe it (see Neiman [3], for example). In this paper, we show that the optimal binary graph is not the Ikeno graph for $n=4$, and for $n>4$ no optimal binary graphs exist. We will give a connection pattern for $2 n$-stage binary graphs which uniquely possess certain optimal properties.

2. Labelings and connection functions for binary graphs. We first give a labeling for the set of vertices of a binary graph. This labeling is useful in specifying the structure of a binary graph, in particular, in determining whether two binary graphs are isomorphic. Let $T$ be a binary tree in $(n+1)$ levels. We label the set of vertices of $T$ except the root as follows:

(i) The set of vertices in the level $k+1, k \geqq 1$, are labeled by the set of $k$-tuples with entries either 0 or 1 , i.e., $\{\mathbf{I}(u): u$ is a vertex in level $k+1$ in $T\}=\{0,1\}^{k}$.

(ii) Let $u$ be a vertex in level $k+1$ adjacent to a vertex $v$ in level $k$. Then, the label of $u, \mathbf{I}(u)$, is either $(\mathbf{I}(v), 0)$ or $(\mathbf{I}(v), 1)$ for $k \geqq 1$.

For a given $2(n+1)$-stage binary graph, we label both the left tree and the right tree satisfying properties (i) and (ii). Then this binary graph can be characterized by a function $f:\{0,1\}^{n} \rightarrow\{0,1\}^{n}$ such that

$$
f\left(a_{1}, \cdots, a_{n}\right)=\left(b_{1}, \cdots, b_{n}\right)
$$

implies that the vertex with label $\left(a_{1}, \cdots, a_{n}\right)$ of the left tree is connected to the vertex with label $\left(b_{1}, \cdots, b_{n}\right)$ of the right tree. $f$ is called the connection function of the binary graph. For example, the connection function of the graph in Fig. 1(a) is $f_{1}(a, b)=(a, b)$. The connection function of the graph in Fig. 1(b) is $f_{2}(a, b)=(b, a)$. Also, the $2(n+1)$-stage Ikeno graph has connection function $f\left(a_{1}, \cdots, a_{n}\right)=\left(a_{n}, \cdots, a_{1}\right)$.

It is easily verified that $f$ is a bijection (one-to-one and onto). For any given bijection $f:\{0,1\}^{n} \rightarrow\{0,1\}^{n}$, we can construct a binary graph having $f$ as its connection function. There are $\left(2^{n}\right)$ ! such bijections. However, two binary graphs constructed by using two different connection functions might be isomorphic. Thus, we need some methods for determining whether two binary graphs with distinct connection functions are isomorphic or not. We first introduce some terminology.

Let $C$ be a vector of $2^{n}-1$ elements; i.e., $C=\left\{c_{i S}: S \subseteq\{1, \cdots, i-1\}\right.$, $1 \leqq i \leqq n\}$, where $c_{i S} \in\{0,1\}$.

Let $\mathbf{C}$ denote the following function from $\{0,1\}^{n}$ to $\{0,1\}^{n}$, such that the $i$ th coordinate of $\mathbf{C}\left(x_{1}, \cdots, x_{n}\right)$ is $x_{i}+\sum s c\{1, \cdots, i-1\} c_{i S} \pi(S)$, where $\pi(S)$ denotes the product of all $x$ in $S$, and $\pi(S)=1$ if $S$ is the empty set. We note that the numbers in the proceeding expression are in $\{0,1\}$ reduced modulo 2 .

LEMMA 1 . Two binary graphs, $G_{1}$ and $G_{2}$, having connection functions $f_{1}$ and $f_{2}$ 
respectively, are isomorphic if and only if there exist two $\left(2^{n}-1\right)$-vectors $C, D$ such that

$$
\mathbf{C} f_{1}=f_{2} \mathbf{D} \text {. }
$$

Proof. Suppose $\mathbf{C} f_{1}=f_{2} \mathbf{D}$ for some vectors $C$ and $D$. We define a map $\alpha$ from the vertex set of $G_{1}$ to the vertex set of $G_{2}$ as follows:

Let $u$ be a vertex of the left tree in $G_{1}$ with label $\left(a_{1}, \cdots, a_{i}\right)$. Then we define $\alpha(u)$ to be the vertex of the left tree in $G_{2}$ with label $\mathbf{D}\left(a_{1}, \cdots, a_{i}\right)$.

Let $v$ be a vertex of the right tree in $G_{1}$ with label $\left(b_{1}, \cdots, b_{i}\right)$. Then we define $\alpha(v)$ to be the vertex of the right tree in $G_{2}$ with label $\mathbf{C}\left(b_{1}, \cdots, b_{i}\right)$.

Moreover, the roots of the left tree and the right tree of $G_{1}$ are mapped to the roots of the left tree and right tree of $G_{2}$ respectively.

It is easily seen that $\alpha$ is one to one and onto. By straightforward verification based on the fact that $\mathbf{C} f_{1}=f_{2} \mathbf{D}$, it can be shown that for $u, v \in V\left(G_{1}\right), u$ is adjacent to $v$ if and only if $\alpha(u)$ is adjacent to $\alpha(v)$ in $G_{2}$. Thus $\alpha$ is an isomorphism, and the two graphs $G_{1}$ and $G_{2}$ are isomorphic.

Now, we assume $G_{1}$ and $G_{2}$ are isomorphic. $f_{1}$ and $f_{2}$ can be viewed as connection functions determined by two labelings $I_{1}$ and $l_{2}$ of the same binary graph $G$. Let us first define another labeling $\mathbf{l}_{3}$ of $G$ such that $\mathbf{l}_{3}(u)=\mathbf{l}_{1}(u)$ for any $u$ in the left tree of $G$ and $\mathbf{l}_{3}(v)=\mathbf{l}_{2}(v)$ for any $v$ in the right tree of $G$. Let $f_{3}$ denote the connection function of $G$ determined by the labeling $\mathbf{l}_{3}$.

Claim. $f_{3}=\mathbf{C} f_{1}$ for some $\left(2^{n}-1\right)$-vector $C$.

Let us first consider all the labelings of $G$ with vertices in the left tree labeled as in $\mathbf{l}_{1}$. There are exactly $2^{2^{n}-1}$ ways to label the right tree. However, for any vector $C=\left\{c_{i S}: S \subseteq\{1, \cdots, i-1\}, 1 \leqq i \leqq n, c_{i S} \in\{0,1\}\right\}$, we can define a labeling of the vertices in the right tree of $G$ as follows:

$$
\mathbf{l}_{C}(v)=\mathbf{C}\left(b_{1}, \cdots, b_{i}\right),
$$

where $\mathbf{l}_{1}(v)=\left(b_{1}, \cdots, b_{i}\right)$.

We note that all labelings $\mathbf{I}_{C}, C \in\{0,1\}^{2^{n}-1}$ are distinct. Thus $\left\{\mathbf{I}_{C}: C \in\{0,1\}^{2^{n}-1}\right\}$ are exactly the set of all labelings of vertices in the right tree of $G$. Therefore, there exists a vector $C$ such that $\mathbf{I}_{3}(v)=\mathbf{l}_{C}(v)$ for any vertex $v$ in the right tree.

Thus, for a terminal vertex $u$ in the left tree which is adjacent to a terminal vertex $v$ in the right tree, we have

$$
\begin{aligned}
& f_{1}\left(\mathbf{l}_{1}(u)\right)=\mathbf{l}_{1}(v), \\
& f_{3}\left(\mathbf{l}_{3}(u)\right)=\mathbf{l}_{3}(v)=\mathbf{l}_{C}(v)=\mathbf{C l}_{1}(v)=\mathbf{C} f_{1}\left(\mathbf{l}_{1}(u)\right) .
\end{aligned}
$$

Since $l_{1}(u)=l_{3}(u)$ for any $u$ in the left tree, we have

$$
f_{3}=\mathbf{C} f_{1} \text {. }
$$

In a similar way it can be shown that $f_{3}=f_{2} \mathbf{D}$ for some vector $D$. Thus we obtain $\mathbf{C} f_{1}=f_{2} \mathbf{D}$ and the lemma is proved.

Lemma 1 is, in fact, equivalent to the following.

Corollary. Two $2(n+1)$-stage binary graphs, $G_{1}$ and $G_{2}$, having connection functions $f_{1}$ and $f_{2}$, respectively, are isomorphic if and only if there exist two $\left(2^{n}-1\right)$-vectors $C$ and $E$ such that

$$
f_{2}=\mathbf{C} f_{1} \mathbf{E} \text {. }
$$

Proof. It suffices to show that the inverse function of the linear transformation $\mathbf{D}$ 
is of the form $\mathbf{E}$ for some vector $E$. This, however, can be done by straightforward calculation to find values of $e_{i j}, 1 \leqq j \leqq i \leqq n$ such that $\mathbf{D E}=\mathbf{I}$ where $\mathbf{I}$ is the identity transformation.

Let $f_{1}, f_{2}$ be two bijections from $\{0,1\}^{n}$ to $\{0,1\}^{n}$. We say $f_{1}$ is equivalent to $f_{2}$ if there exists two $\left(2^{n}-1\right)$-vectors, say $C$ and $D$ such that

$$
f_{1}=\mathbf{C} f_{2} \mathbf{D} \text {. }
$$

We note that two connections functions derived from different labelings of a binary graph are equivalent.

3. The optimal 8-stage binary graph. It can be easily verified that the Ikeno graph is an optimal $2 n$-stage binary graph for $n=1,2$ and 3 . However, as an immediate result of the following theorem the Ikeno graph is not optimal for $n=4$.

THEOREM 1. The optimal 8-stage binary graph $G_{8}$ is isomorphic to the binary graph determined by the connection function $f\left(a_{1}, a_{2}, a_{3}\right)=\left(a_{3}, a_{2}+a_{1} a_{3}, a_{1}\right)$ as shown in Fig. 2.

Before we prove Theorem 1, we state a result of Takagi [6]. Let $T_{k}$ denote the class of 4-stage channel graphs where there are $k$ each of the second-stage and the third-stage vertices, and each second-stage (third-stage) vertex is connected to two third-stage (second-stage) vertices.

Theorem (Takagi). A graph in $T_{k}$ is optimal if and only if the $2 k$ middle-stage links form a cycle.

Proof of Theorem 1. Let $L_{i}$ denote the set of $i$ th stage links. It suffices to show that in each of the following four cases, the blocking probability of $G_{8}$ achieves minimum (since the four cases are mutually disjoint and exhaustive).

(i) Both links in $L_{1}$ and both links in $L_{7}$ are idle. In this case, an 8-stage binary graph is not blocked if there exists a path from any second-stage vertex to any seventh-stage vertex. Therefore the graph can be viewed as a 6-stage graph by eliminating the first and last stage and combining vertices in stage 2 and stage 7 . By shrinking a graph from stage $i$ to stage $j$ we mean replacing every path between the two stages by a link. When all these paths are edge disjoint, then by simply defining the probability of the new link being busy as the probability of the path being busy, the blocking probability of the shrunken graph is the same as the blocking probability of the original graph. By shrinking the reduced $G_{8}$ from stage 2 to stage 5 , we obtain a 4-stage graph in $T_{4}$. Fig. 3 shows such a $T_{4}$ obtained from $G_{8}$ :

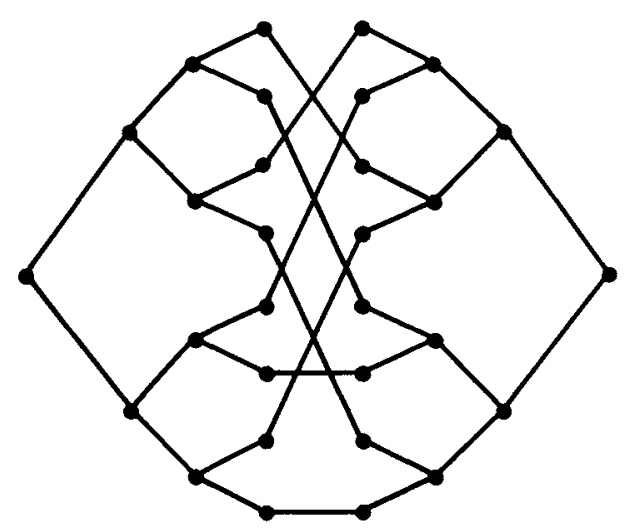

Fig. 2 


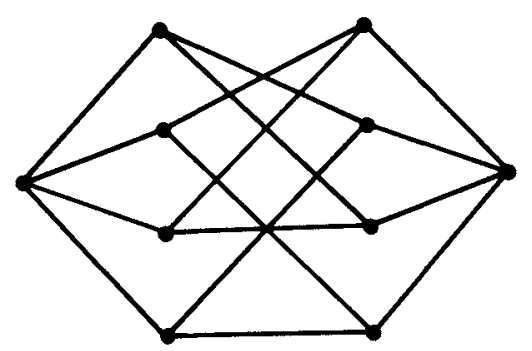

FiG. 3

Note that the eight middle-stage links in this graph form a cycle. Theorem 1 follows immediately from the Takagi Theorem.

(ii) Both links in $L_{1}$ and one link in $L_{7}$ are idle. $G_{8}$ is reduced to the 6-stage graph in Fig. 4. It is straightforward to verify that this is the best reduction.

(iii) One link in $L_{1}$ and both links in $L_{7}$ are idle. Same as (ii).

(iv) One link in $L_{1}$ and one link in $L_{7}$ are idle. We have to consider the set of four reduced graphs corresponding to the four possible combinations of idle links. There are three possible sets for 8-stage binary graphs. A Type A set consists of two graphs of four paths and two empty graphs. A Type B set consist of two graphs of three paths and two graphs of one path. A Type C set consists of four graphs of two paths. The reduced $G_{8}$ is a Type $C$ set and it is straightforward to verify that the sum of blocking probabilities of the four graphs in a Type $\mathrm{C}$ set is minimal.

From (i), (ii), (iii) and (iv), we conclude that $G_{8}$ is an optimal 8-stage graph. It is straightforward to verify that $G_{8}$ is indeed the unique optimal 8-stage graph up to isomorphism since there are only a few distinct connections to check. We omit the details here.

The 8-stage Ikeno graph $I_{8}$ is illustrated in Fig. 5 . It can be easily seen that $G_{8}$ is superior to the 8-stage Ikeno graph $I_{8}$ since the blocking probability of $G_{8}$ is less than or equal to the blocking probability of $I_{8}$ in case (i); and the blocking probability of $G_{8}$ is equal to the blocking probability of $I_{8}$ in case (ii), (iii) and (iv) for any given link occupancies.

4. Some preliminary results on binary graphs. In a channel graph $G$, let $u$ be a vertex in stage $i$ and $v$ a vertex in stage $j$ where $j>i$. The channel subgraph determined by $u$ and $v$ in $G$ is defined to be the union of all paths of length $j-i$ connecting $u$ and $v$ in $G$. A maximal channel subgraph of $G$ is a channel subgraph of $G$ having the number of stages one less than the number of stages in $G$. A maximal channel subgraph is usually determined by a vertex in the second stage and the sink (the vertex in the last stage) or the source (the vertex in the first stage) and a vertex in the stage next to the last.

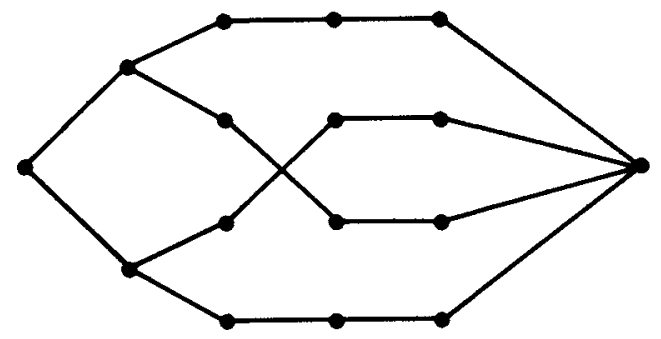

FIG. 4 


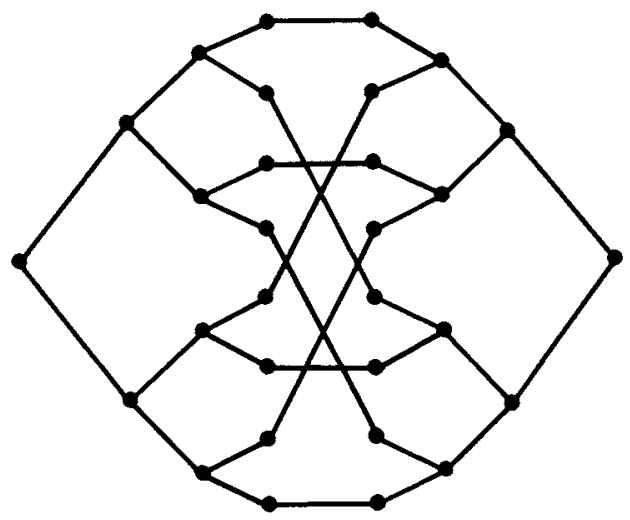

FIG. 5

We say a maximal channel subgraph of a $2 n$-stage binary graph is optimal if it isomorphic to an optimal $2(n-1)$-stage binary graph after shrinking from stage $n-1$ to stage $n+1$ or from stage $n$ to stage $n+2$.

LEMMA 2. All the maximal channel subgraphs of an optimal $2 n$-stage binary graph are optimal.

Proof. Let $G^{\prime}$ be a maximal channel subgraph determined by a vertex in stage 2 and the sink in the optimal $2 n$-stage binary graph $G$. Let $H$ be a $2 n$-stage binary graph such that all its maximal channel subgraphs determined by a vertex in stage 2 and the sink are, after shrinking from stage $n$ to stage $n+2$, isomorphic to a given arbitrary $2(n-1)$-stage binary graph $H^{\prime}$. To be precise, let $f$ denote the connection function of a $2(n-1)$-stage binary graph. We can then construct $H$ with the connection function $f^{*}$ to be $f^{*}\left(a_{1}, \cdots, a_{n-1}\right)=\left(b_{1}, \cdots, b_{n-1}\right)$, where $f\left(a_{2}, \cdots, a_{n-1}\right)=\left(b_{1}, \cdots\right.$, $b_{n-2}$ ) and $a_{1}=b_{n}$. Now we consider the link occupancies $p_{1}, \cdots, p_{n-1}$ where $p_{1}$ is extremely small compared with $p_{i}, i \neq 1$. Then the blocking probabilities of $G$ and $H$ can be approximated by the product of $p_{1}$ and the blocking probability of $G^{\prime}$ and $H^{\prime}$, respectively. Since $G$ is an optimal binary graph, $G^{\prime}$ must be optimal.

COROLLARY. If no optimal $2 n$-stage binary graph exists, then no optimal $2 \mathrm{~m}$ stage binary graph exists for $m \geqq n$.

In a later section we will show that optimal 10-stage binary graphs do not exist. Thus optimal $2 n$-stage binary graphs, $n \geqq 5$, do not exist. It is then of interest to investigate binary graphs with certain optimal properties.

We note that the optimal $2 n$-stage binary graphs, for $n \leqq 4$, have the symmetric property, i.e., the connection function $f$ satisfies the following:

$$
\begin{aligned}
& f\left(a_{1}, \cdots, a_{n-1}\right)=\left(b_{1}, \cdots, b_{n-1}\right), \text { if and only if } \\
& f\left(b_{1}, \cdots, b_{n-1}\right)=\left(a_{1}, \cdots, a_{n-1}\right) .
\end{aligned}
$$

In other words, the connection function $f$ is idempotent, $f=f^{-1}$. Symmetry might be a desirable property for practical considerations since it facilitates the construction and the control of the network.

We define $G_{8}$ to be an HS-optimal 8-stage binary graph (it is in fact optimal). For $n \geqq 5$, a $2 n$-stage binary graph is said to be hereditary if all its maximal channel subgraphs, after shrinking, are $2(n-1)$-stage HS-optimal binary graphs. We call a $2 n$ stage binary graph HS-optimal if it is both hereditary and symmetric. The main result of this paper is to prove that for $n \geqq 5$, there exists a unique HS-optimal $2 n$-stage 
binary graph. In this section, we give a set of lemmas concerning HS-optimal $2 n$ stage binary graphs.

From the definition of HS-optimal binary graphs, the following observations are immediate.

LEMMA 3. Let $f$ be the connection function of an HS-optimal $2(n+1)$-stage binary graph. We define the function $\bar{f}_{a_{1}}:\{0,1\}^{n-1} \rightarrow\{0,1\}^{n-1}$ by the following:

$$
\bar{f}_{a_{1}}\left(a_{2}, \cdots, a_{n}\right)=\left(b_{1}, \cdots, b_{n-1}\right),
$$

where $f\left(a_{1}, a_{2}, \cdots, a_{n}\right)=\left(b_{1}, \cdots, b_{n}\right)$. Then $\bar{f}_{a_{1}}$ is the connection function of the maximal channel subgraph determined by the sink and the vertex of the left tree with labeling $\left(a_{1}\right)$. Similarly $\left(\bar{f}^{-1}\right)_{b_{1}}$ is the connection function of the maximal channel subgraph determined by the source and the vertex of the right tree with labeling $\left(b_{1}\right)$.

LEMMA 4. A $2(n+1)$-stage binary graph having connection function fis hereditary if and only if $\bar{f}_{a}$ and $\left(\bar{f}^{-1}\right)_{b}$, for $a, b \in\{0,1\}$, are equivalent to connection functions of an HS-optimal $2 n$-stage binary graph.

Proof. This follows from the definition of hereditary.

LEMMA 5. Let $f$ be the connection function of an HS-optimal $2(n+1)$-stage binary graph $G$. Then $f$ is equivalent to the following function $g$ :

$$
g\left(a_{1}, \cdots, a_{n}\right)=\left(\bar{f}_{a_{1}}\left(a_{2}, \cdots, a_{n}\right), a_{1}\right) .
$$

Proof. It suffices to show that we can choose a proper labeling of $G$ with connection function $g$. Because $G$ is HS-optimal, for any labeling I of $G$ the two vertices with labeling $\left(b_{1}, \cdots, b_{n-1}, 0\right)$ and $\left(b_{1}, \cdots, b_{n-1}, 1\right)$ are connected to two vertices in stage $n+1$ with distinct first components, i.e.,

$$
f\left(a_{1}, \cdots, a_{n}\right)=\left(b_{1}, \cdots, b_{n-1}, 0\right)
$$

and

$$
f\left(a_{1}^{\prime}, \cdots, a_{n}^{\prime}\right)=\left(b_{1}, \cdots, b_{n-1}, 1\right)
$$

imply $a_{1} \neq a_{1}^{\prime}$.

Thus we consider a labeling $\mathrm{I}^{\prime}$ of $G$ such that a vertex $v$ with $\mathbf{I}(v)=$ $\left(b_{1}, \cdots, b_{n-1}, b_{n}\right)$ will be labeled as $\mathrm{I}^{\prime}(v)=\left(b_{1}, \cdots, b_{n-1}, b_{n}+1\right)$, if $a_{1} \neq b_{n}$, and $\mathbf{l}^{\prime}(v)=\left(b_{1}, \cdots, b_{n}\right)$ otherwise, where $f\left(a_{1}, \cdots, a_{n}\right)=\left(b_{1}, \cdots, b_{n}\right)$. We note that the connection function $g$ under the labeling $l^{\prime}$ can be written as follows:

$$
g\left(a_{1}, \cdots, a_{n}\right)=\left(\bar{f}_{a_{1}}\left(a_{2}, \cdots, a_{n}\right), a_{1}\right) .
$$

Since $f$ and $g$ are connection functions of $G$ in two labelings, we know $f$ and $g$ are equivalent from Lemma 2.

Thus Lemma 5 is proved.

LEMMA 6. Let $x$ be a number depending on a where $x, a \in\{0,1\}$. Then $x$ can be written as follows:

$$
x=a w_{1}+w_{2} \quad(\bmod 2),
$$

where $w_{1}, w_{2}$ are some constants in $\{0,1\}$.

Proof. It is easy to find $w_{1}, w_{2}$ by solving two equations for $a=0$ or 1 . Namely, $w_{2}=x(0), w_{1}=x(1)-x(0)$.

For any vector $C=\left\{c_{i S}\right\}$ we let $\mathbf{C}_{a}$ denote the linear transformation determined by $\left\{c_{i S} a\right\}$.

LEMMA 7. Let $C$ be a $\left(2^{n}-1\right)$-vector $\left\{c_{i S}: S \subseteq\{1, \cdots, i-1\}, i=1, \cdots, n\right\}$ where $c_{i s}$ 's depend on a. Then the linear transformation $\mathbf{C}$ can be written as follows: 


$$
\mathbf{C}=\mathbf{D E}=\mathbf{F G}
$$

where $E$ and $F$ are $\left(2^{n}-1\right.$ )-vectors (independent of $a$ ) and $D=D_{a}, G=G_{a}$.

Proof. The values of elements of E, and $\mathbf{D}$ can be obtained by using Lemma 6 and solving the $n(n+1)$ linear equations derived from $\mathbf{C}=\mathbf{D E}$. The values of elements of $\mathbf{F}$ and $\mathbf{G}$ can be obtained similarly.

LEMMA 8. Let $G$ be a $2(n+1)$-stage hereditary binary graph. There exists a labeling of $G$ such that the connection function can be written as follows:

$$
f\left(a_{1}, \cdots, a_{n}\right)=\left(\mathbf{M}_{a_{1}} g \mathbf{N}_{a_{1}}\left(a_{2}, \cdots, a_{n}\right), a_{1}\right),
$$

where $g$ is some connection function of an HS-optimal $2 n$-stage binary graph, and $n_{i \phi}=0$, for $i=1, \cdots, n-1$.

Proof. Let $h$ be the connection function of a $2(n+1)$-stage hereditary binary graph $G$. From Lemma 5 we may assume

$$
h\left(a_{1}, \cdots, a_{n}\right)=\left(b_{1}, \cdots, b_{n}\right)=\left(\bar{h}_{a_{1}}\left(a_{2}, \cdots, a_{n}\right), a_{1}\right) .
$$

Since $G$ is hereditary, we know that, by Lemma $4, \bar{h}_{a_{1}}\left(a_{2}, \cdots, a_{n}\right)=$ $\left(b_{1}, \cdots, b_{n-1}\right)$ is a connection function of the $2 n$-stage HS-optimal binary graph. By the induction assumption, we have $\bar{h}_{a}=\mathbf{R} g \mathbf{S}$ where the elements of $R$ or $S$ depend on $a$. Therefore, by Lemma 7 , we have

$$
\mathbf{R}=\mathbf{U M}_{a},
$$

and

$$
\mathbf{S}=\mathbf{N}_{a}^{\prime} \mathbf{V}
$$

where elements of $U$ and $V$ are independent of $a$. We define $U^{\prime}$ and $V^{\prime}$ such that $\mathbf{U}^{\prime}\left(x_{1}, \cdots, x_{n}\right)=\left(\mathbf{U}\left(x_{1}, \cdots, x_{n-1}\right), x_{n}\right) \mathbf{V}^{\prime}\left(x_{1}, \cdots, x_{n}\right)=\left(x_{1}, \mathbf{V}\left(x_{2}, \cdots, x_{n}\right)\right)$, and we note that we can find a $\left(2^{n}-1\right)$-vector $W$ such that $\mathbf{W}\left(x_{1}, \cdots, x_{n}\right)=$ $\left(x_{1}, \mathbf{W}_{x_{i}}^{\prime}\left(x_{2}, \cdots, x_{n}\right)\right)$ and $\mathbf{N}_{a_{1}}^{\prime}=\mathbf{N}_{a_{1}} \mathbf{W}_{a_{1}}^{\prime}$, where $n_{i \phi}=0$ for $i=1, \cdots, n$.

It is easy to verify that

$$
\mathbf{U}^{\prime} f \mathbf{W} \mathbf{V}^{\prime}=h,
$$

where $f\left(a_{1}, \cdots, a_{n}\right)=\left(\mathbf{M}_{a_{1}} g \mathbf{N}_{a_{1}}\left(a_{2}, \cdots, a_{n}\right), a_{1}\right)$. Therefore, Lemma 8 is proved.

From now on, for any HS-optimal binary graph, we will only consider its connection functions in form as described in Lemma 8. We note that several examples of connection functions we give in this paper are in this form.

LEMMA 9. A $2(n+1)$-stage binary graph $G$ is HS-optimal if and only if the connection function of $G$ is equivalent to $f$ satisfying

$$
\begin{aligned}
f\left(a_{1}, \cdots, a_{n}\right) & =\left(\mathbf{M}_{a_{1}} g \mathbf{N}_{a_{1}}\left(a_{2}, \cdots, a_{n}\right), a_{1}\right) \\
& =\left(a_{n},\left(\mathbf{N}_{b_{2}}\right)^{-1} g^{-1}\left(\mathbf{M}_{t_{n}}\right)^{-1}\left(a_{1}, \cdots, a_{n-1}\right)\right),
\end{aligned}
$$

where $g$ is a connection function of the optimal $2 n$-stage binary graph and $M$ and $N$ are two $\left(2^{n}-1\right)$-vectors.

Proof. Since $G$ is symmetric and hereditary, we have (1) from Lemma 8 . Now, suppose $G$ has a connection function equivalent to $f$. It follows immediately $G$ is symmetric. By Lemma 3 and $4, G$ is also hereditary. Thus $G$ is HS-optimal.

Now we consider a set of connection functions $P=\left\{p_{i}: i=1,2, \cdots\right\}$ where $p_{i}:[0,1]^{i} \rightarrow[0,1]^{i}$. We set 


$$
\begin{aligned}
p_{2}\left(a_{1}, a_{2}\right) & =\left(a_{2}, a_{1}\right), \\
p_{3}\left(a_{1}, a_{2}, a_{3}\right) & =\left(a_{3}, a_{2}+a_{1} a_{3}, a_{1}\right) \quad(\bmod 2) .
\end{aligned}
$$

The $p_{i}$ for $i \geqq 4$, will be defined in $\S \S 5,6$.

We note that $p_{2}, p_{3}$ are connection functions for optimal 6- and 8-stage binary graphs. The set $P$ denotes the set of the connection functions for HS-optimal binary graphs.

5. 10-stage binary graphs. For 10-stage binary graphs, we will show the following.

THEOREM 2. The HS-optimal 10-stage binary graph has connection function (up to isomorphism) as follows (see Fig. 6):

$$
p_{4}\left(a_{1}, a_{2}, a_{3}, a_{4}\right)=\left(a_{4}, a_{3}+a_{2} a_{4}, a_{2}+a_{1} a_{3}+a_{1} a_{2} a_{4}, a_{1}\right) \quad(\bmod 2) .
$$

We remind the reader that all calculations are in $Z_{2}$. We will, from now on, omit the notation $(\bmod 2)$.

Proof. It is straightforward to verify that $p_{4}$ is idempotent.

The function $p_{4}$ can be written as

$$
\begin{aligned}
p_{4}\left(a_{1}, a_{2}, a_{3}, a_{4}\right) & =\left(\mathbf{L}_{a_{1}} p_{3}\left(a_{2}, a_{3}, a_{4}\right), a_{1}\right) \\
& =\left(a_{4}, p_{3} \mathbf{L}_{a_{4}}\left(a_{1}, a_{2}, a_{3}\right)\right),
\end{aligned}
$$

where

$$
\begin{aligned}
\mathbf{L}_{a}\left(x_{1}, x_{2}, x_{3}\right) & =\left(x_{1}, x_{2}, x_{3}+a x_{2}\right) \\
& =\left(\mathbf{L}_{a}\right)^{-1}\left(x_{1}, x_{2}, x_{3}\right) .
\end{aligned}
$$

It follows from Lemma 9 that the binary graph having $p_{4}$ as the connection function is HS-optimal.

Now, suppose $G$ is an HS-optimal 10-stage binary graph having connection function $f$. From Lemma $9 f$ satisfies the following

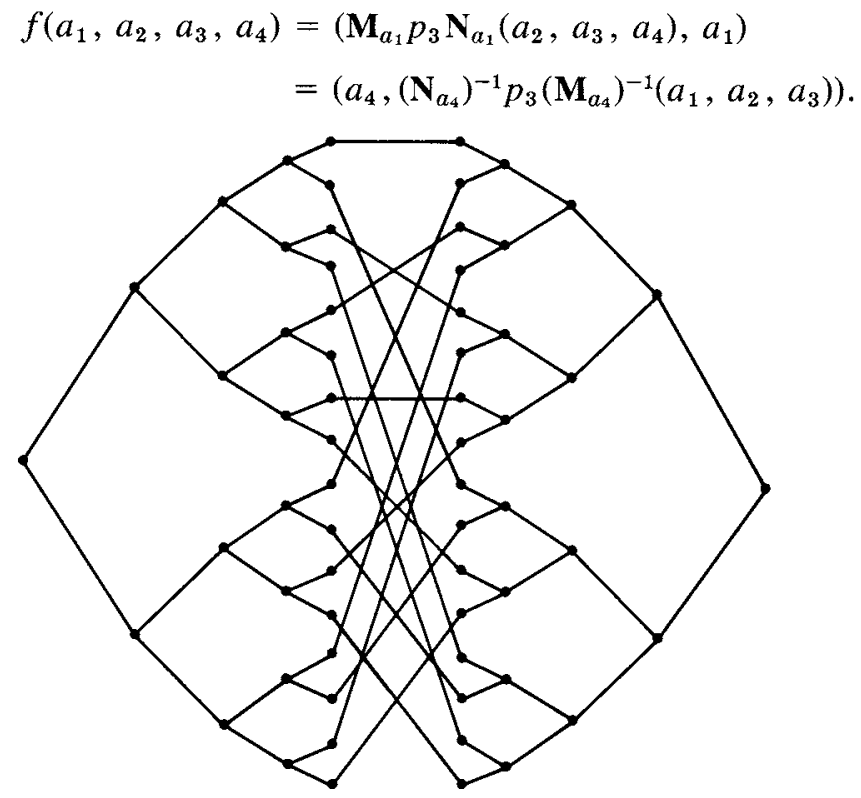

FIG. 6 
To simplify the notation we denote the sets $\{1\},\{2\},\{1,2\}$ by $1,2,12$, respectively. Equating the values in the first coordinates of (2) and (3), we obtain

$$
\begin{array}{r}
a_{4}=a_{4}+a_{1} a_{2} n_{3,1}+a_{1} a_{3} n_{3,2}+a_{1} a_{2} a_{3} n_{3,12}+a_{1} m_{1 \phi}, \\
\text { for any } a_{i} \in\{0,1\}, 1 \leqq i \leqq 4 .
\end{array}
$$

Thus, we have $m_{1 \phi}=n_{3,1}=n_{3,2}=n_{3,12}=0$.

Suppose we replace $a_{4}$ by 0 . The value of the second coordinates in (2) and (3) is

$$
a_{3}=a_{3}+m_{2 \phi} a_{1}+n_{2,1} a_{1} a_{2} .
$$

Therefore $m_{2 \phi}=n_{2,1}=0$ and $\mathbf{N}$ is then the identity function. Let us write

$$
\mathbf{M}\left(x_{1}, x_{2}, x_{3}\right)=\left(x_{1}, x_{2}+m_{2,1} x_{1}, x_{3}+m_{3 \phi}+m_{3,1} x_{1}+m_{3,2} x_{2}+m_{3,12} x_{1} x_{2}\right) .
$$

Then we have

$$
\begin{aligned}
& \mathbf{M}^{-1}\left(x_{1}, x_{2}, x_{3}\right)=\left(x_{1}, x_{2}+m_{2,1} x_{1}, x_{3}+m_{3 \phi}\right. \\
& \left.\quad+\left(m_{3,1}+m_{2,1} m_{3,2}+m_{2,1} m_{3,12}\right) x_{1}+m_{3,2} x_{2}+m_{3,12} x_{1} x_{2}\right) .
\end{aligned}
$$

We consider the value of the second coordinates in (2) and (3). We have

$$
\begin{gathered}
a_{3}+a_{2} a_{4}+m_{2,1} a_{1} a_{4}=a_{3}+m_{3 \phi} a_{4}+\left(m_{3,1}+m_{2,1} m_{3,2}+m_{2,1} m_{3,12}\right) a_{4} a_{1} \\
+m_{3,2} a_{2} a_{4}+m_{3,12} a_{1} a_{2} a_{4} .
\end{gathered}
$$

Therefore we have $m_{3,1}=m_{3 \phi}=m_{3,12}=0, m_{3,2}=1$.

Now we consider the third coordinate in (2) and (3). We have $a_{2}+a_{1} a_{3}+$ $a_{1} a_{2} a_{4}=a_{2}+m_{2,1} a_{1} a_{4}+a_{1} a_{3}+a_{1} a_{2} a_{4}$. Therefore we have $m_{2,1}=0, \mathbf{M}=\mathbf{L}$ and $f=p_{4}$. Theorem 2 is proved.

It should be noted that the 10-stage HS-optimal binary graph is not optimal since in the case that $p_{1}=p_{2}=p_{8}=p_{9}=0$, then the graph can be reduced and shrunken to a 4-stage graph in $T_{8}$ (similar to what we did to $G_{8}$ in the proof of Theorem 1 ). However, the 16 middle-stage links form two cycles instead of one. Since there does exist a 10-stage binary graph which, after similar reductions and shrinking, yields a graph in $T_{8}$ with the middle-stage links forming a cycle, then by Takagi's Theorem the HS-optimal 10-stage binary graph is not optimal. Furthermore, it can be shown (by straightforward arguments) that there exists only one (up to isomorphism) hereditary graph which is not symmetric. Again by using Takagi's Theorem it can be seen that this graph is not optimal. However, by Lemma 2, an optimal 10-stage binary graph must be hereditary. Therefore, no optimal 10-stage binary graph exists.

6. $2 n$-stage binary graphs for $n \geqq 5$. The main result in this paper is the following.

THEOREM 3. The HS-optimal $(2 n+2)$-stage binary graph has the connection function (up to isomorphism) as follows:

$$
p_{n}\left(a_{1}, \cdots, a_{n}\right)=\left(b_{1}, \cdots, b_{n}\right),
$$

where

$$
\begin{aligned}
& b_{1}=a_{n}, \\
& b_{i}=a_{n+i-1}+a_{n-i} b_{i-1} \quad(\bmod 2), \text { for } i=2, \cdots, n-1, \\
& b_{n}=a_{1} .
\end{aligned}
$$


Proof. First we define

$$
\mathbf{L}\left(x_{1}, \cdots, x_{i}\right)=\left(x_{1}, \cdots, x_{i-1}, x_{i}+x_{i-1}\right) .
$$

It can be verified by induction that

$$
\begin{aligned}
p_{n}\left(a_{1}, \cdots, a_{n}\right) & =\left(\mathbf{L}_{a_{1}} p_{n-1}\left(a_{2}, \cdots, a_{n}\right), a_{1}\right) \\
& =\left(a_{n}, p_{n-1} \mathbf{L}_{a_{n}}\left(a_{1}, \cdots, a_{n-1}\right)\right) .
\end{aligned}
$$

The binary graph determined by $p_{n}$ is thus symmetric and hereditary.

Now we consider a connection function $g$ of HS-optimal $2(n+1)$-stage binary graph $G$. From Lemmas 8 and $9, g$ is equivalent to an $f$ which has the following form

$$
\begin{aligned}
f\left(a_{1}, \cdots, a_{n}\right) & =\left(\mathbf{M}_{a_{1}} p_{n-1} \mathbf{N}_{a_{1}}\left(a_{2}, \cdots, a_{n}\right), a_{1}\right) \\
& =\left(a_{n},\left(\mathbf{N}_{a_{n}}\right)^{-1} p_{n-1}\left(\mathbf{M}_{a_{n}}\right)^{-1}\left(a_{1}, \cdots, a_{n-1}\right)\right) .
\end{aligned}
$$

We want to show by induction on $n$ that:

(*): $\quad$ if $f$ can be written in the form of (4) and (5), then $\mathbf{N}$ is the identity mapping and $\mathbf{M}=\mathbf{L}$.

(*) is true for $n=4$ from Theorem 2. Suppose (*) is true for all $n^{\prime}$ with $4 \leqq n^{\prime}<n$. We will prove that $(*)$ holds for $n$.

The value in the first coordinates in (4) and (5) is

$$
a_{n}=a_{n}+\sum_{S \subseteq\{0, \cdots, n-1\}} c_{n, S} \pi(S), \quad \text { for all } a_{i} \in\{0,1\}, 1 \leqq i \leqq n .
$$

Thus we have $c_{n, S}=0$, for all $S$.

We consider, $\mathbf{M}^{\prime}, \mathbf{N}^{\prime}$ and $\mathbf{H}$ to be mappings from $\{0,1\}^{n-2} \rightarrow\{0,1\}^{n-2}$ such that

$$
\begin{aligned}
\mathbf{M}\left(0, x_{2}, \cdots, x_{n-1}\right) & =\left(0, \mathbf{M}^{\prime}\left(x_{2}, \cdots, x_{n-1}\right)\right), \\
\mathbf{N}\left(x_{1}, \cdots, x_{n-1}\right) & =\left(\mathbf{N}^{\prime}\left(x_{1}, \cdots, x_{n-2}\right), x_{n-1}\right), \\
\mathbf{H}\left(x_{1}, \cdots, x_{n-2}\right) & =\left(x_{1}+m_{21}, x_{2}+m_{31}, \cdots, x_{n-2}+m_{n-1,1}\right) .
\end{aligned}
$$

It is easy to see that

$$
\begin{aligned}
\mathbf{M}\left(x_{1}, \cdots, x_{n-1}\right) & =\left(x_{1}, \mathbf{H}_{x_{1}} \mathbf{M}^{\prime}\left(x_{2}, \cdots, x_{n-1}\right)\right), \\
\mathbf{M}^{-1}\left(x_{1}, \cdots, x_{n-1}\right) & =\left(x_{1}, \mathbf{M}^{\prime-1} \mathbf{H}_{x_{1}}\left(x_{2}, \cdots, x_{n-1}\right)\right), \\
\mathbf{N}^{-1}\left(x_{1}, \cdots, x_{n-1}\right) & =\left(\mathbf{N}^{\prime-1}\left(x_{1}, \cdots, x_{n-2}\right), x_{n-1}\right) .
\end{aligned}
$$

By induction $p_{n-1}\left(x_{1}, \cdots, x_{n-1}\right)=\left(x_{n-1}, p_{n-2} \mathbf{L}_{x_{n-1}}\left(x_{1}, \cdots, x_{n-2}\right)\right)$. Therefore (4) is equal to the following:

$$
\begin{aligned}
& \left(\left(\mathbf{M}_{a_{1}}\left(a_{n}, p_{n-2} \mathbf{L}_{a_{n}} \mathbf{N}_{a_{1}}^{\prime}\left(a_{2}, \cdots, a_{n-1}\right)\right)\right), a_{1}\right) \\
& \quad=\left(a_{n}, \mathbf{H}_{a_{1} a_{n}} \mathbf{M}_{a_{1}}^{\prime} p_{n-2} \mathbf{L}_{a_{n}} \mathbf{N}_{a_{1}}^{\prime}\left(a_{2}, \cdots, a_{n-1}\right), a_{1}\right) .
\end{aligned}
$$

On the other hand, (5) is equal to the following:

$$
\begin{aligned}
\left(a_{n}, \mathbf{N}_{a_{n}}^{-1}\left(\mathbf{L}_{a_{1}} p_{n-2} \mathbf{M}_{a_{n}}^{\prime-1} \mathbf{H}_{a_{1} a_{n}}\left(a_{2}, \cdots, a_{n-1}\right), a_{1}\right)\right) \\
\quad=\left(a_{n}, \mathbf{N}_{a_{n}}^{-1} \mathbf{L}_{a_{1}} p_{n-2} \mathbf{M}_{a_{n}}^{\prime-1} \mathbf{H}_{a_{1} a_{n}}\left(a_{2}, \cdots, a_{n-1}\right), a_{1}\right) .
\end{aligned}
$$

Therefore we have

$$
\mathbf{H}_{a_{1} a_{n}} \mathbf{M}_{a_{1}}^{\prime} p_{n-2} \mathbf{L}_{a_{n}} \mathbf{N}_{a_{1}}^{\prime}=\mathbf{N}_{a_{n}}^{-1} \mathbf{L}_{a_{1}} p_{n-2} \mathbf{M}_{a_{n}}^{\prime-1} \mathbf{H}_{a_{1} a_{n}} .
$$


By setting $a_{n}=0$ in (6), we have

$$
\mathbf{M}_{a}^{\prime} p_{n-2} \mathbf{N}_{a_{1}}^{\prime}=\mathbf{L}_{a} p_{n-2} .
$$

From the above equality and the definition of $p_{n-2}$ and $\mathbf{L}$ we define $f^{\prime}$ as follows:

$$
\begin{aligned}
f^{\prime}\left(x_{1}, \cdots, x_{n-1}\right) & =\left(\mathbf{M}_{x_{1}}^{\prime} p_{n-2} \mathbf{N}_{x_{1}}^{\prime}\left(x_{2}, \cdots, x_{n-1}\right), x_{1}\right) \\
& =\left(\mathbf{L}_{x_{1}} p_{n-2}\left(x_{2}, \cdots, x_{n-1}\right), x_{1}\right) \\
& =\left(x_{n-1}, p_{n-2} \mathbf{L}_{x_{n-1}}\left(x_{1}, \cdots, x_{n-2}\right)\right) \\
& =\left(x_{n-1}, \mathbf{N}_{x_{n-1}}^{-1} p_{n-1} \mathbf{M}_{x_{n-1}}^{\prime-1}\left(x_{1}, \cdots, x_{n-2}\right)\right) .
\end{aligned}
$$

By the induction assumptions, we have $\mathbf{M}_{x}^{\prime}=\mathbf{L}_{x}$ and $\mathbf{N}_{x}^{\prime}$ is the identity mapping. Therefore $\mathbf{N}$ is the identity mapping and (6) is equivalent to the following:

$$
\mathbf{H}_{a_{1} a_{n}} \mathbf{L}_{a_{1}} p_{n-2} \mathbf{L}_{a_{n}}=\mathbf{L}_{a_{1}} p_{n-2} \mathbf{L}_{a_{n}} \mathbf{H}_{a_{1} a_{n}} .
$$

By setting $a_{1}=a_{n}=a$ in (7), we have

$$
\mathbf{H}_{a} \mathbf{L}_{a} p_{n-2} \mathbf{L}_{a}=\mathbf{L}_{a} p_{n-2} \mathbf{L}_{a} \mathbf{H}_{a},
$$

i.e.,

$$
\mathbf{H}_{a} \mathbf{L}_{a} p_{n-2} \mathbf{L}_{a} \mathbf{H}_{a} \mathbf{L}_{a}=\mathbf{L}_{a} p_{n-2} .
$$

Now we consider $f^{\prime \prime}$ as follows:

$$
\begin{aligned}
f^{\prime \prime}\left(x_{1}, \cdots, x_{n-1}\right) & =\left(\mathbf{H}_{x_{1}} \mathbf{L}_{x_{1}} p_{n-2} \mathbf{L}_{x_{1}} \mathbf{H}_{x_{1}} \mathbf{L}_{x_{1}}\left(x_{2}, \cdots, x_{n-1}\right), x_{1}\right) \\
& =\left(\mathbf{L}_{x_{1}} p_{n-2}\left(x_{2}, \cdots, x_{n-1}\right), x_{1}\right) \\
& =\left(x_{n-1}, p_{n-2} \mathbf{L}_{x_{n-1}}\left(x_{1}, \cdots, x_{n-2}\right)\right) \\
& =\left(x_{n-1}, \mathbf{L}_{x_{n-1}} \mathbf{H}_{x_{n-1}} \mathbf{L}_{x_{n-1}} p_{n-2} \mathbf{L}_{x_{n-1}} \mathbf{H}_{x_{n-1}}\left(x_{1}, \cdots, x_{n-2}\right)\right) .
\end{aligned}
$$

As before, we have, by the induction assumptions, $\mathbf{H}_{a} \mathbf{L}_{a}=\mathbf{L}_{a}$ and $\mathbf{L}_{a} \mathbf{H}_{a} \mathbf{L}_{a}$ is the identity. This implies $\mathbf{H}_{a}$ is the identity and $\mathbf{M}=\mathbf{L}$. Therefore we have

$$
\begin{aligned}
f\left(a_{1}, \cdots, a_{n}\right) & =\left(\mathbf{L}_{a_{1}} p_{n-1}\left(a_{2}, \cdots, a_{n}\right), a_{1}\right)=\left(a_{n}, p_{n-1} \mathbf{L}_{a_{n}}\left(a_{1}, \cdots, a_{n-1}\right)\right) \\
& =\left(b_{1}, \cdots, b_{n}\right) .
\end{aligned}
$$

Theorem 3 is then proved.

7. Construction of switching networks with prescribed channel graphs. We will present several constructions of switching networks with prescribed channel graphs.

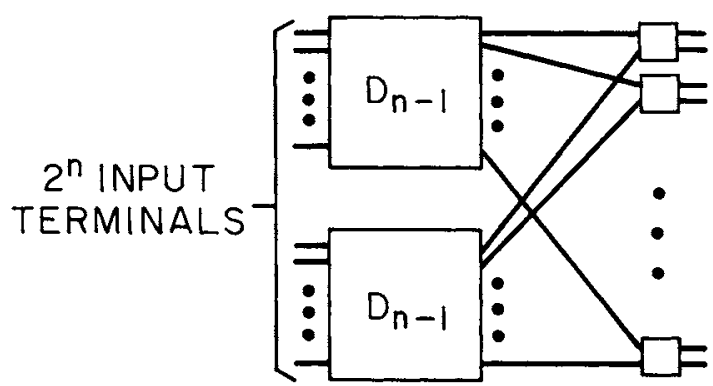

FIG. 7 


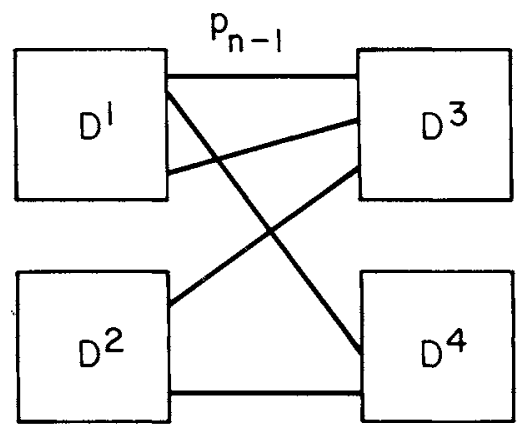

FIG. 8

Let us first consider a $2 n$-stage network $N_{2 n}$ consisting of $2 \times 2$ switches. Let $D_{1}$ denote a $2 \times 2$ switch and $D_{n}$ be the network built recursively as shown in Fig. 7 . The $2 n$-stage network $N_{2 n}$ consists of 2 copies of $D_{n}$ denoted by $D^{1}, D^{2}$ together with 2 copies denoted by $D^{3}$ and $D^{4}$, of the mirror image of $D_{n}$ as shown in Fig. 8.

The linking pattern between the middle two stages is determined by the connection function of the channel graph $p_{n-1}$. Namely, the ith output switch of $D_{1}$ or $D_{2}$ is connected to the $j$ th input switch of $D^{3}$ or $D^{4}$ if and only if $i-1$ and $j-1$ have the binary expressions $\left(a_{1}, \cdots, a_{n-1}\right),\left(b_{1}, \cdots, b_{n-1}\right)$ respectively, and $p_{n-1}\left(a_{1}, \cdots, a_{n-1}\right)=\left(b_{1}, \cdots, b_{n-1}\right)$.

It can be easily verified that the channel graph of $N_{2 n}$ is the HS-optimal binary graph $G_{2 n}$.

Let us now consider networks with an odd number of stages. We note that a binary graph of $2 n+2$ stages can be viewed as a binary graph of $2 n+1$ stages by shrinking the two middle stages. We can then derive HS-optimal binary graphs $G_{2 n+1}$ from the HS-optimal graphs $G_{2 n+2}$. For example $G_{5}$ is as shown in Fig. 9.

Now, we want to construct a $2 n+1$ stage network with channel graph $G_{2 n+1}$. $G_{2 n+1}$ consists of a copy of $D_{n+1}$ together with two copies, denoted by $D^{3}, D^{4}$, of the mirror image of $D_{n}$ as shown in Fig. 10.

The linking pattern between stage $n+1$ and stage $n+2$ is determined by $p_{n}$. Namely, the $i$ th output switch of $D_{n+1}$ is connected to the $j$ th input line of $D^{3}$ or $D^{4}$ if and only if $i-1$ and $j-1$ have the binary expressions $\left(a_{1}, \cdots, a_{n}\right),\left(b_{1}, \cdots\right.$, $\left.b_{n}\right)$, respectively, and $p_{n}\left(a_{1}, \cdots, a_{n}\right)=\left(b_{1}, \cdots, b_{n}\right)$. It is easily verified that $N_{2 n+1}$ has channel graph $G_{2 n+1}$.

8. Concluding remarks. We can generalize the ideas in this paper and consider the connection pattern of two $t$-ary graphs. A $(2 n+2)$-stage Ikeno graph is the $t$-ary graph determined by the connection function $f\left(x_{1}, \cdots, x_{n}\right)=\left(x_{n}, \cdots, x_{1}\right)$

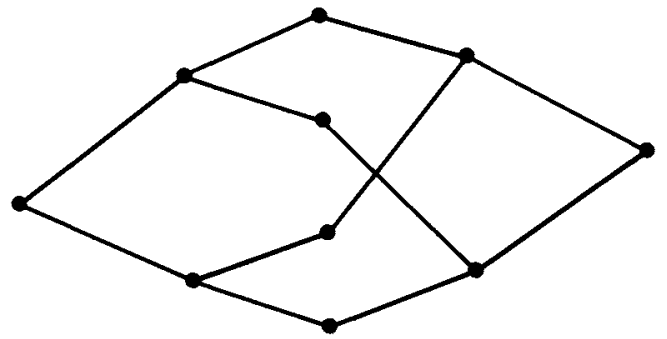

FiG. 9 


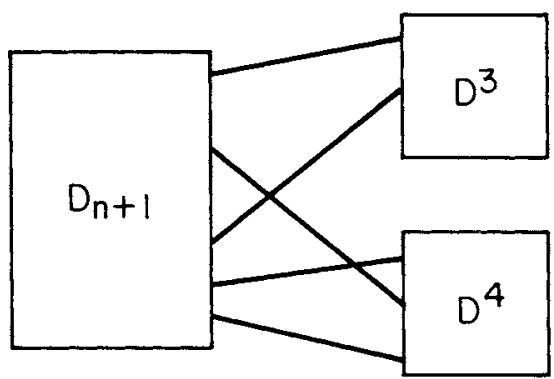

FIG. 10

where the leaves of the left and right $t$-ary trees are labeled by elements in $\{0, \cdots, t-1\}$. Again it can be shown that the Ikeno graph is not optimal for $t \geqq 3$, $n \geqq 3$ by considering an alternative connection function defined in a similar way as we defined $p_{n}$ in previous sections. The connection patterns of $t$-ary graphs are probably more complicated than the binary case.

\section{REFERENCES}

[1] N. IKENO, A limit on crosspoint number, IRE Trans. Inform. Theory, Vol. IT-5 (1959), pp. 187-196.

[2] P. LEGALL, Etude du blocade dans les systèmes de commutation téléphonique, Ann. Télécommun., 11 , No. 9 (1956).

[3] V. I. NEIMAN, Structural properties of connecting networks, $8^{\text {th }}$ ITC, Milbourne (1976), pp. 131-1 to 131-8.

[4] K. TAKAGI, Design of multi-stage link systems by means of optimal channel graphs, Electronics and Communications in Japan, Vol. 51-A (1968), pp. 37-46.

[5] ___ Optimum channel graph oflink system, Electronics and Communications in Japan, Vol. 54-A (1971), pp. 1-10.

[6] - A comparison of channel graphs for link system design, Trans. IECE Japan, Vol. E61, No. 7 (1978), pp. 538-539. 